schuss, es gebe wenig Zweifel daran, dass Telemedizin zu einer besseren Vernetzung der Versorgung beitragen könne. Er mahnte die Verhandlungspartner im Bewertungsausschuss, wie vom Gesetzgeber gefordert, zu einer akzeptablen Einigung über die Erstattung der Telemedizin zu kommen: „Bei diesem Thema wird seit einer Dekade herumgewabert. Das ist nicht zu akzeptieren". Koschorrek befürchtet, dass die Entscheidung des Bewertungsausschusses auf den kleinsten gemeinsamen Nenner hinauslaufen könnte, auf beispielsweise eine sehr niedrig dotierte Abrechnungsziffer.

Was aus ihrer Sicht nötig ist, machten die Vertreter der Telemedizinbranche deutlich: Für monatliche Beträge zwischen 50 und $100 €$ könne Telemonitoring in unterschiedlicher Abstufung angeboten werden. Alles darunter gehe auf Kosten der Qualität.

Philipp Grätzel von Grätz

\title{
Online-Terminbuchung: Techniker Krankenkasse startet bundesweite Förderstudie
}

\author{
Mit $120 €$ unterstützt die Techniker Krankenkasse Ärzte, die bei ihrem \\ einjährigen Feldversuch zur Online-Terminbuchung mitmachen. Sämtliche \\ Arztgruppen sind angesprochen.
}

$\mathrm{N}$ ach einem ersten Pilotversuch, an dem 90 Berliner Fachärzte und 800 TK-Versicherte teilnahmen, will die Techniker Krankenkasse (TK) jetzt in großem Stil testen, ob und was es bringt, Arzttermine online zu buchen. Die Resonanz aus dem ersten Lauf sei ausgesprochen gut gewesen, heißt es. Einer Befragung zufolge hätten die meisten Ärzte (85\%) das Verfahren als Arbeitserleichterung empfunden. Die meisten

\section{Teilnahmebedingungen \\ Die genauen Teilnahmebedingungen sowie ausführliche Erläuterungen zum Ablauf der TK-Förderstudie "Online-Terminbuchung":}

\section{http://bit.ly/YcAilO}

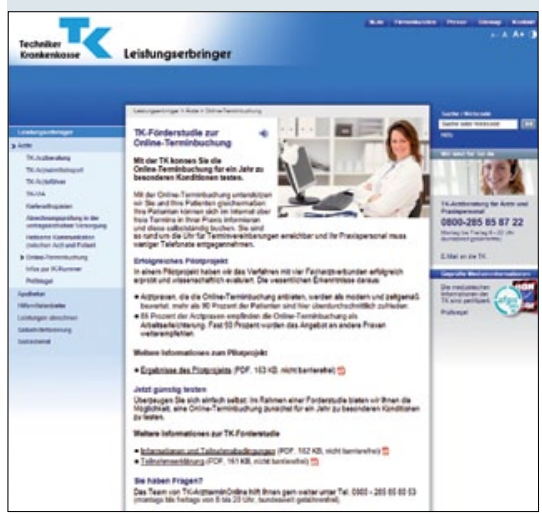

Patienten (über $90 \%$ ) zeigten sich zufrieden mit der Wartezeit auf einen Termin und würden die Online-Buchung wahrscheinlich wiederholt in Anspruch nehmen.

Ging es in der Bundeshauptstadt noch darum, auszuprobieren, ob die OnlineBuchung bei Fachärzten mit besonders enger Terminlage Entlastung schafft, so soll jetzt bundesweit und über sämtliche Arztgruppen hinweg beobachtet werden, welche Effekte eine regional umfassendere Abdeckung hat. Dazu sind entsprechend viele Teilnehmer nötig. Als Anreiz lockt die TK Kassenärzte mit einer finanziellen Unterstützung von $120 €$. Bedingung ist, dass sie über die gesamte Laufzeit (vom 1. Juni 2013 bis 31. Mai 2014) bei der Förderstudie mitmachen sowie an der begleitenden Evaluation in Form zweier Befragungen.

In der Praxis sieht die Sache so aus: Die Basisdaten des Arztes werden zusammen mit seiner Online-Terminbuchung mit dem TK-Portal www.tk.de/ terminbuchung verlinkt. Dort können Patienten - und zwar nicht nur TK-Versicherte - sich dann Termine reservieren. Teilnehmende Ärzte, heißt es, könnten selbst entscheiden, wie viele Termine sie online anbieten. Auch gebe es keine Verpflichtung, TK-Versicherte bei der Terminvergabe zu bevorzugen.

Christoph Winnat

\section{Warnung vor digitaler Lösegelderpressung}

Das Bundeskriminalamt (BKA) warnt vor Schadsoftware, die den Rechner von betroffenen Computeranwendern sperrt und eine Art Lösegeld einfordert. Wenn ein PC infiziert ist, erscheint auf dem Bildschirm ein nicht weg zu klickendes Fenster mit dem Logo des BKA, so die Behörde. Dabei werde behauptet, dass die Funktion des Computers „aus Gründen unbefugter Netzaktivitäten ausgesetzt" sei. In dem Fenster stehe beispielsweise, dass "die Wiedergabe von pornografischen Inhalten mit Minderjährigen festgestellt" worden sei. Das BKA ist nicht Urheber der Meldung. Die Betroffenen sollen laut BKA auf keinen Fall auf den Erpressungsversuch eingehen. Der Nutzer werde aufgefordert, $100 €$ über die Zahlungsdienstleister uKash oder Paysafecard zu zahlen, damit der Rechner entsperrt wird. Der Rechner sei in diesem Fall aber schon infiziert und werde auch durch eine Zahlung des Lösegelds nicht wieder sauber.

\section{Windows-8-Nachfolger im Netz aufgetaucht}

Im Netz ist eine Vorabversion des nächsten Windows veröffentlicht worden, berichten das US-Portal "The Verge" sowie andere Websites. Windows 8 ermögliche es, mehrere App-Fenster nebeneinander anzuzeigen. Außerdem soll das Betriebssystem "Windows Blue" eine stärkere Personalisierung erlauben. Kleinere oder größere Kachelsymbole für einzelne Apps auf dem Desktop sollen möglich sein. Es wird erwartet, dass Windows Blue in den nächsten Monaten zum Testen verfügbar ist und eine offizielle Version bis Jahresende vorliegt. Das polnische Blog "Winforum.eu“ habe Screenshots veröffentlicht.

\section{Neue Tools für die Do-It-Yourself-Homepage}

Die $1 \& 1$ Internet AG hat ihre Do-lt-Yourself-Homepage um etliche Features erweitert, die helfen sollen, Informationen für Kunden und Website-Besucher ansprechender und professioneller aufzubereiten. Dazu gehören laut $1 \& 1$ das „1\&1 Multimedia-Show Tool", mit dem man audiovisuelles Material für die Kundenansprache gestalten könne sowie das „1\&1 Online Broschüren Tool“ zur Erstellung von pdf-Broschüren mit Bild und Text. Siehe auch www.1und1.info. 